\title{
Expediente
}

A Revista Palíndromo é uma publicação do Programa de Pós-Graduação em Artes Visuais do Centro de Artes da Universidade do Estado de Santa Catarina. Existe desde 2004, inicialmente na forma impressa e depois apenas em modo eletrônico a partir de 2009. Trata-se de uma revista digital sem fins lucrativos e concebida para ser um veículo de divulgação de pesquisas e produção de conhecimento, devidamente inscrita na plataforma do Sistema Eletrônico de Editoração de Revistas (SEER).

Palíndromo é uma palavra de origem grega que indica o que pode ser lido numa direção e também no sentido inverso, ou seja, de trás para frente. Avessa à ordem e às normas pré-estabelecidas, a pesquisa em/ sobre artes visuais remete não apenas a normas negadas, como demanda constante revisão de dados, processos e reorganização de ideias, acolhendo o que pode ser pensado como trânsito e travessia que desconhece uma só direção.

\section{ISSN 21752346}

Volume 12, número 27, maio - agosto 2020

UNIVERSIDADE DO ESTADO DE SANTA CATARINA - UDESC

Reitor: Prof. Dr. Dilmar Baretta

\section{CENTRO DE ARTES - CEART}

Diretora Geral: Prof. Dra. Maria Cristina da Rosa Fonseca da Silva

DEPARTAMENTO DE ARTES VISUAIS - DAV

Chefe: Prof. Dra. Raquel Stolf

PROGRAMA DE PÓS-GRADUAÇÃO EM ARTES VISUAIS - PPGAV

Coordenadora: Prof. Dra. Jociele Lampert de Oliveira

\section{EDITORA-CHEFA}

Mara Rúbia Sant'Anna

\section{EDITORAS DA PALÍNDROMO 2020 -2022}

Luana Wedekin

Mara Rúbia Sant'Anna

Sandra Maria Correa Fávero 


\section{CORPO EDITORIAL TÉCNICO}

Elisa V. Queiroz

Gabriel Bonfim

Gabriela Gonzaga V. Rodrigues

Marcel Angelo Timon Frias

Miguel Vassali

Pablo Paniagua

CONSELHO DE PARECERISTAS - Palíndromo v.12, n.27, Mai - Ago 2020

Alena Rizi Marmo Jahn (Univille)

Alexandre Pedro de Medeiros (Unicamp)

Amanda Saba Ruggiero (USP)

Ana Lucia Beck (UFRGS)

Angelica Oliveira Adverse (UFMG)

Bettina Rupp (UFRN)

Bruna Wulff Fetter (UFRGS)

Christiane Pereira Arcuri (UFRJ)

Chrystianne Goulart Ivanóski (UFSC)

Cláudia Mariza Mattos Brandão (UfPel)

Cláudio Tarouco de Azevedo (UfPel)

Clediane Lourenço

Clelia Maria Lima de Mello (UFSC)

Daniela Kern (UFRGS)

Denise Bandeira (UNESPAR)

Eloisa da Rosa Oliveira (UFSC)

Eunice Maria da Silva (USP)

Fabíola Alves (Unesp)

Gabriela Kremer Motta (UfPel)

Isabel Almeida Carneiro (UERJ)

Jéssica Becker

Jose Carlos Carlos Rocha (UDESC)

Katia Prates (UFRGS)

Katyuscia Sosnowski (IFPR)

Luana M. Wedekin (UDESC)

Luiz Sérgio da Cruz de Oliveira (UFF)

Manoela dos Anjos Afonso Rodrigues (FAV/UFG)

Marcos H. Camargo (UNESPAR)

Mário de Faria Carvalho (UFPE)

Priscila Leonel (UNESP)

Regilene Aparecida Sarzi-Ribeiro (UNESP)

Sandra Mónica Figueiredo Oliveira (FBA/UP)

Thiago Costa (IFMT)

Vera Lucia Didonet Thomaz (UFRGS)

Wilson Roberto da Silva (UNIFESSPA)

Yasmin Fabris (UFPR) 
CONSELHO DE PARECERISTAS DAS EDIÇÕES DE 2018 A 2019 - listagem complementar Ana Saldanha (UNESP)

Ana Valéria de Figueiredo (UERJ)

André Pitol (USP)

André Winter Noble (UFRGS)

Anna Amelia Faria (BAHIANA)

Antonio Carlos Vargas (UDESC)

Bernadette Panek (UNESPAR/EMBAP)

Bianca Scliar (UDESC)

Cleomar de Sousa Rocha (UFG)

Elaine Schmidlin (UDESC)

Fabio Wosniak (UDESC)

Fellipe Eloy Teixeira Albuquerque (SMESP)

Flávia Pedrosa Vasconcelos (UFSM)

Gabriel Augusto de Paula Bonfim (UDESC)

Germana Konrath (UFRGS)

Gilvânia Maurício Dias de Pontes (UFRGS)

Gustavo Cunha Araujo (UFT)

Leandro Alves Garcia (UFRN)

Lislaine Sirsi Cansi (UfPel)

Luan Sevignani (UniTrento)

Luciane Ruschel N. Garcez (UDESC)

Luciano Pessoa (USP/UNIP)

Maria Salete Borba (UNICENTRO)

Marina Bortoluz Polidoro (UFRGS)

Nadia da Cruz Senna (UfPel)

Natália de Noronha Santucci (UFRGS)

Patricia Carlesso Marcelino (UPF)

Paulo Ivan Rodrigues Vega Júnior (UnB)

Ramsés Albertoni Barbosa (IAD-UFJF)

Rodrigo Luis dos Santos (UNISINOS)

Rodrigo Montandon Born (UDESC)

Rogério Rosa Rodrigues (UDESC)

Sandra Margarete Abello (Unoesc)

Simone Rocha da Conceição (UFRGS)

Sônia Tramujas Vasconcellos (Unespar)

Valeska Bernardo Rangel (IFSC)

Viviane Baschirotto (UDESC)

Yara Rondon Guasque Araujo (UDESC)

DIAGRAMAÇÃO

Gabriela Gonzaga V. Rodrigues

Miguel Vassali 


\section{CONTATO}

revistapalindromo@udesc.br

Palíndromo [recurso eletrônico] / Universidade do Estado de Santa Catarina. Centro de Artes. Programa de Pós-Graduação em Artes Visuais. v. 1, n. 1, (2004) - . - Florianópolis : UDESC/CEART, 2004 -

\section{Quadrimestral}

ISSN-e 2175-2346

DOI $10.5965 / 21752346$

Disponivel em: <www.revistas.udesc.br/index.php/palindromo/index> Palíndromo (acesso em 10 set. 2019).

A partir de 2009 a revista passa existir apenas em modo eletrônico.

1. Artes plásticas. 2. Arte - Estudo e ensino - periódicos. I. Universidade do Estado de Santa Catarina. Centro de Artes.

CDD: $707-20$. ed.

Ficha catalográfica elaborada pela Bibliotecário Orestes Trevisol Neto CRB 14/1530 Biblioteca Central da UDESC 